\title{
Review of Potential for Precision Electroweak Studies at the LHC
}

Dominique Pallin on behalf of the ATLAS and CMS collaborations

\section{Introduction}

A big effort has been made by the ATLAS and CMS collaborations to demonstrate the very high discovery potential offered at the LHC collider. Nevertheless, as well as D0 and CDF collaborations at the Tevatron, the LHC collaborations have also a large potential to perform precision measurements in the electroweak sector.

The Standard Model reproduces well all the electroweak measurements done so far. However some caveats in the electroweak global fit could indicate that there is still something to understand, leaving some room for improved measurements of some of the electroweak observables. Among them, the $W$ mass, the Top mass and the effective weak mixing angle $\sin ^{2} \theta_{\text {eff }}^{l}$ are the most important electroweak parameters to be measured more precisely in future experiments.

$m_{t o p}$ in one hand, $M_{W}$ and $\sin ^{2} \theta_{e f f}^{l}$ on the other hand play a different role in the Standard Model. Three accurate input parameters are used to fix the Standard Model: $M_{Z}$ the $\mathrm{Z}$ boson mass, $G_{\mu}$ the muon decay constant and $\alpha$ the electromagnetic coupling constant. In addition to a small set of parameters $\left(m_{l}, m_{q}\right.$ including $m_{t o p}, \alpha_{s}$ and $\left.M_{H}\right)$, they are used to calculate the Standard Model prediction of all electroweak observables. Taking into account the radiative corrections, $M_{W}$ and $\sin ^{2} \theta_{\text {eff }}^{l}$ have the largest sensitivity to the Higgs mass. Then, because the Top mass enters as an input parameter, the predictions for $M_{W}$ and $\sin ^{2} \theta_{\text {eff }}^{l}$ depend also on it. Even if the Top mass is known with a better precision than the other quark masses, its experimental uncertainty is the dominant contribution to the parametric error on the Standard Model prediction of $M_{W}$ and $\sin ^{2} \theta_{\text {eff }}^{l}$.

A better measurement of the Top mass, in addition to more precise measurements of $M_{W}$ and $\sin ^{2} \theta_{e f f}^{l}$ (both having a different sensitivity to the Top and Higgs masses), could lead to a deeper understanding of the Standard Model, a more precise indirect derivation of the Higgs mass, and could give a better sensitivity to search for deviation from the Standard Model. 


\subsection{EW Precision measurements: past and future}

The current experimental precision on $M_{W}$ is $34 \mathrm{MeV}$ [1] (Fig. 1), mainly driven by the Tevraton results on transverse $\mathrm{W}$ mass reconstruction [2], and by LEP 2 measurements where $\mathrm{W}$ pairs events are fully reconstructed [1].

The top mass has been directly measured by D0 and CDF at Tevatron RunI. The average of the two measurements leads to a precision of $5.1 \mathrm{GeV}$ [3]. (Fig. 1). For what concern $\sin ^{2} \theta_{e f f}^{l}$, the various measurements lead to a

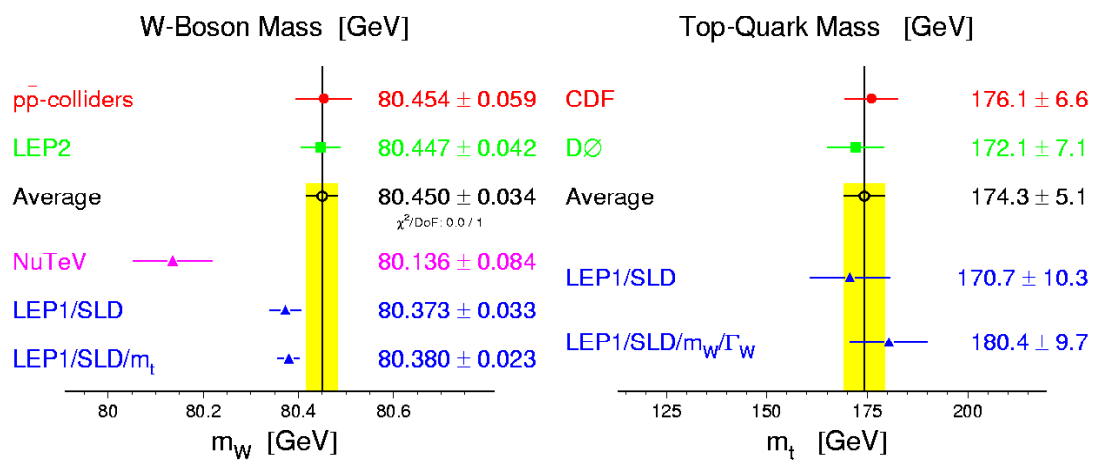

Fig. 1. $M_{W}$ and $m_{t o p}$ mass measurements

current precision of $17.10^{-5}$ [1] (Fig. 2). A new and precise mesurement would give interesting informations to understand the potential discrepancy existing between the measurement from LR asymmetry at SLD and the measurement from $b$ quark forward-backward asymmetry at LEP.

Using all available data, the Standard Model fit constraints the Higgs mass with a $50 \%$ relative uncertainty [1]; $\sin ^{2} \theta_{\text {eff }}^{l}$ carrying the dominant weight, even if the $\mathrm{W}$ mass is known with a better relative precision. New measurements of these crucial EW parameters will come in the future. The Tevatron $p-\bar{p}$ collider is in Run2 phase [4]. By 2007, CDF and D0 could expect to collect $15 \mathrm{fb}^{-1}$ at $\sqrt{s}=1.96 \mathrm{TeV}$, two orders of magnitude more than during Run1 phase. The LHC $p-p$ collider will start in 2007 [5]. The high luminosity expected at LHC, $10^{33} \mathrm{~cm}^{-2} \mathrm{~s}^{-1}$ (low luminosity phase) rising to $10^{34} \mathrm{~cm}^{-2} \mathrm{~s}^{-1}$ (high luminosity phase), together with the large center of mass energy, $\sqrt{s}=14 \mathrm{TeV}$, will provide to ATLAS [6] and CMS [7] a large event rate, much larger than for the Tevatron Run2. It is expected to collect $10 \mathrm{fb}^{-1}$ per year during the low luminosity phase and up to $100 \mathrm{fb}^{-1}$ in the high luminosity phase. 


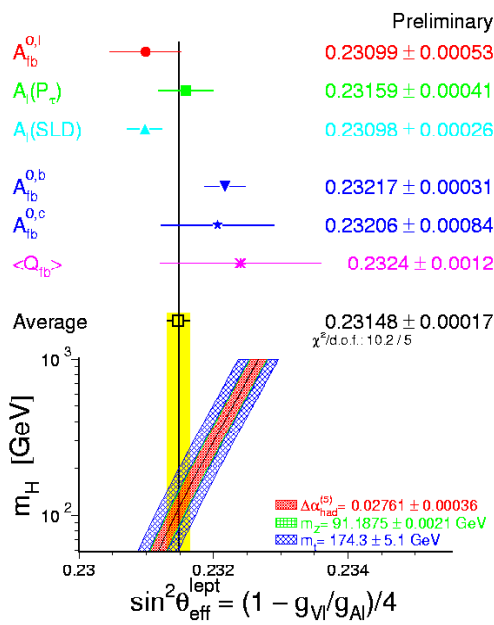

Fig. 2. The effective weak mixing angle current measurements

\section{Precision EW measurements at $\mathrm{LHC}$}

\subsection{Production cross sections and detectors parameters}

After one year of LHC running at low luminosity, the expected number of events for processes involving EW measurements is very high: $10^{8} \mathrm{~W}$ leptonic decays events and $10^{7}$ events for $t \bar{t}$ production (Table 1 ). This huge potential statistics is essential to perform precision measurements. With such a large event rate, the statistical error on EW precision measurements will be negligible, large samples for calibration and control of the systematics will help to reduce significantly the systematic errors. Nevertheless, in addition to the physical background present at high rate $\left(10^{9}\right.$ inclusive jets with $P_{T}>200 \mathrm{GeV}$ per year of running at low lum.), pile-up (2.3 minimum bias events per crossing at low lum. and 10 times more at high lum.) will also have a potential impact on the achievable precision measurements. For this reason, most of the EW precision measurements will be performed during the low luminosity phase. The precision on the $\mathrm{W}$ and Top mass measurements will be mainly driven by the performances reached on the lepton and jet energy absolute calibrations. The goals are challenging : $0.02 \%$ precision for the lepton scale and $1 \%$ for the jet scale. The lepton energy and momentum scale will be determined $i n$-situ using the large available sample of $Z \rightarrow l l$ decays. The challenge is that several sub-detectors are concerned, in addition to the needed precise knowledge of various detector ingredients (material to $1 \%$, alignement to $1 \mu \mathrm{m}$, magnetic field maps to $0.1 \%$...). Preliminary studies [8] indicate that the foreseen precision could be achieved. The standard way to determine the jet scale is to perform a $P_{T}$ balance in $\mathrm{Z}+$ jet $(Z \rightarrow l l)$ or $\gamma+$ jet events. At LHC, the light jet calibration will also be performed using very 
Table 1. Cross section and approx. number of events for some key processes at LHC in one year of running at Low luminosity

\begin{tabular}{lcc}
\hline Process & $\sigma(\mathrm{pb})$ & Events/year \\
\hline $\mathrm{W} \rightarrow e \nu$ & $1.510^{4}$ & $10^{8}$ \\
$\mathrm{Z} \rightarrow e^{+} e^{-}$ & $1.510^{3}$ & $10^{7}$ \\
$t \bar{t}$ & 833 & $10^{7}$ \\
$b \bar{b}$ & $510^{8}$ & $10^{12}$ \\
\hline
\end{tabular}

clean sample of $W \rightarrow j j$ selected in the lepton+jet channel of $t \bar{t}$ decays [8] [9]. It might be also possible to determine the b-jet energy scale to the level of $1 \%$ precision using $Z \rightarrow b \bar{b}$ decays.

\subsection{Top Mass measurement at the LHC}
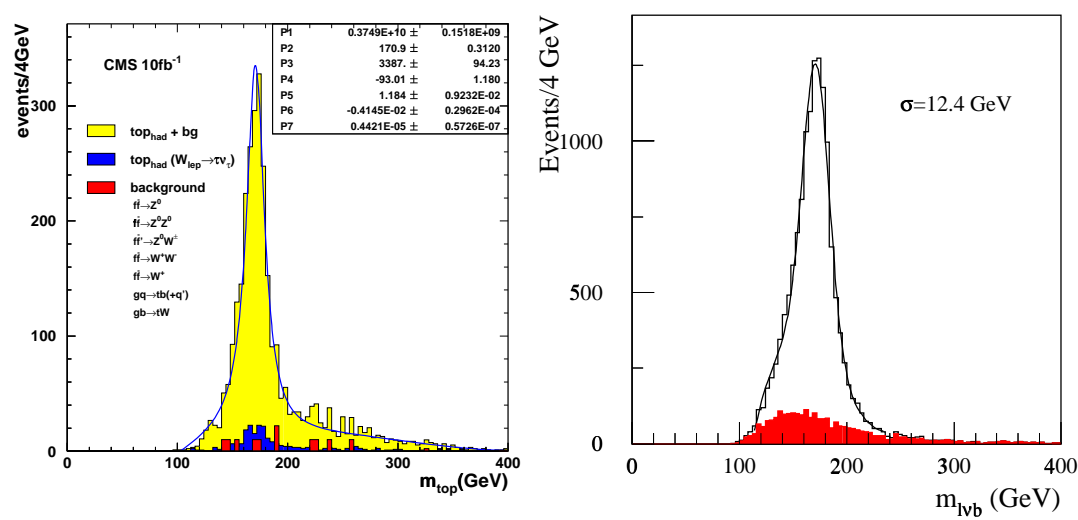

Fig. 3. left: Invariant mass of the $t \rightarrow b j j$ decay products(CMS) with the background from physics processes (dark area).

right: Invariant mass of the $t \rightarrow b l \nu$ decay products(ATLAS) with the contribution from the combinatorial background (dark area). The neutrino momentum is determined from the missing transverse energy and the $\mathrm{W}$ mass constraint

With 8 millions of $t \bar{t}$ events produced in one year at low luminosity, the LHC will be an excellent place to study the Top quark properties . The best channel to measure the Top mass is the lepton+jet channel, where one W decays hadronically, the other $\mathrm{W}$ decaying into $l \nu$. The presence of a high $P_{T}$ lepton with two b jets allows a clean tagging of these events which represents $30 \%$ of the $t \bar{t}$ decays. Several methods have been investigated to determine 
the Top mass from the lepton+jet channel [9] [10] [11] [12]. A first method is to extract the Top mass from the invariant mass of the hadronic part $(t \rightarrow W b \rightarrow j j b)$ (Fig. 3 left). A sample of 45000 events could be selected per year at low luminosity, $70 \%$ being well reconstructed $t \bar{t}$ events, the remaining ones comming essentially from wrong combinations of jets in the $t \bar{t}$ decay. With such a large sample, the statistical error on $m_{T_{o p}}$ is negligible $(0.1$ $\mathrm{GeV}$ ). The dominant systematic errors come from the b-jet energy scale (the light-jet energy scale being constrained in situ by the decay $W \rightarrow j j$ ) and FSR effects.

A second approach takes advantage of the special topology of high $P_{T}$ $\left(P_{T}>200 \mathrm{GeV}\right) t \bar{t}$ pairs. In this case, the two Top quarks are produced back to back, the decay products of the hadronic top being merged in a global calorimeter cluster, and the invariant mass computed from the individual calorimeter cells over a given cone around the Top direction. Even if the sample is smaller (5600 events), the statistical error is still small $(0.2 \mathrm{Gev})$, and the Top mass is sensitive to a different set of systematic errors (Table 2c) with respect to the previous method.

Table 2. Systematic error on the Top mass determination, per exp., per year of running at low lum, for various channels $(l=e, \mu)$

\begin{tabular}{|c|c|c|c|c|}
\hline Channel & $\begin{array}{c}\mathrm{a} \\
W_{1} \rightarrow q \bar{q} \\
W_{2} \rightarrow q \bar{q}\end{array}$ & $\begin{array}{c}\mathrm{b} \\
W_{1} \rightarrow l \nu \\
W_{2} \rightarrow l \nu\end{array}$ & $\begin{array}{c}\mathrm{c} \\
W_{1} \rightarrow q \bar{q} \\
W_{2} \rightarrow l \nu \\
\text { high } P_{T} \text { sample }\end{array}$ & $\begin{array}{c}\mathrm{d} \\
W_{1} \rightarrow q \bar{q} \\
W_{2} \rightarrow l \nu \\
\text { full sample }\end{array}$ \\
\hline stat & 0.2 & 0.3 & 0.2 & 0.1 \\
\hline b-jet scale $(1 \%)$ & 0.7 & 0.7 & & 0.7 \\
\hline FSR & 2.8 & 0.6 & 0.1 & 0.5 \\
\hline light-jet scale & 0.8 & & & 0.2 \\
\hline Comb. BKG & & & & 0.1 \\
\hline b frag & 0.3 & 0.7 & 0.3 & 0.1 \\
\hline ISR & 0.4 & 0.1 & 0.1 & 0.1 \\
\hline $\mathrm{PDF}$ & & 1.2 & & \\
\hline Calo. cells calib/noise & & & 2.2 & \\
\hline total $\Delta m_{T o p}(G e V)$ & 3 & 1.7 & 2.3 & 0.9 \\
\hline
\end{tabular}

An other method has been developped to reduce further the systematic uncertainties. It makes use of the whole information by performing a kinematical fit of the entire $t \bar{t}$ semileptonic decay, reconstructing the neutrino from the missing transverse energy and the $\mathrm{W}$ mass constraint(Fig. 3 right). The $\chi^{2}$ of the fit helps to separate combinatorial background from right combinations of $t \bar{t}$ decay products in one hand, and allows also to reduce the impact of poorly reconstruced jets due to effect arising from FSR and semi-leptonic 
decays of b-quark jets. In that case, assuming that a precision of $1 \%$ on the b-jet scale is reached by ATLAS and CMS, a precision of $1 \mathrm{GeV}$ on the Top mass determination is possible (Table $2 \mathrm{~d}$ ).

Fully leptonic and fully hadronic top decays channels have been also investigated. The studies have shown that after only one year of data taking at low luminosity, a total error on the Top mass at the level of 2-3 GeV can be achieved for these channels (Table 2a,b).

\subsection{W Mass measurement at the LHC}

The Top mass and the $\mathrm{W}$ mass give an equal weight in the global $\mathrm{EW}$ fit if the experimental precision of the measurements are related by $\Delta M_{W}=$ $0.007 \Delta m_{T o p}$. The expected precision achievable at LHC on the Top mass gives the precision to reach on the $\mathrm{W}$ mass, of the order of $15 \mathrm{MeV}$. The methods foreseen to determine the $\mathrm{W}$ mass are the same as at Tevatron Run2. But LHC will benefit from the very large statistics available in $\mathrm{W}$ and $\mathrm{Z}$ decays, about 60 millions for $\mathrm{W}$ and 6 millions for $\mathrm{Z}$ decays per year at low luminosity and per experiment.

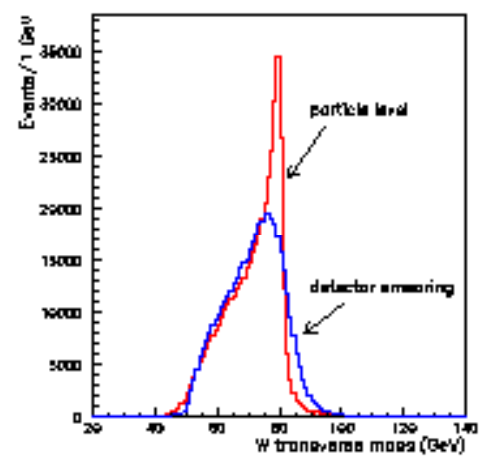

Fig. 4. Distribution of the $\mathrm{W}$ transverse mass as obtained at particle level and by including the expected ATLAS detector resolution

The best method for the low luminosity phase is to extract the $\mathrm{W}$ mass from the transverse mass of the $W \rightarrow l \nu$ decay, where the experimental distribution (Fig. 4) is fitted to Monte-Carlo samples with different values of $M_{W}$ [13] . The statistical error being being very small because of the large available decays, the main uncertainties will come for the Monte-Carlo modelling of the physics ( knowledge of the $P_{T}^{W}$ spectrum, structure functions, $\mathrm{W}$ width, $\mathrm{W}$ radiative decays and background) and the detector response (lepton energy/momentum resolution and absolute scale, response to recoil). Most of the systematics will be driven by the number of observed $\mathrm{Z}$ decays. PDF will be also constrained by LHC data. As shown on the Table 3, 
the knowledge of the absolute lepton energy/momentum scale will be the dominant uncertainty. In order to be at the same level as for the other systematics, the absolute scale should be known with a $0.02 \%$ accuracy. The total expected error on the $\mathrm{W}$ mass measurement, for one lepton family, per experiment and per year of data taking at low luminosity, is presented on Table 3. Most of the systematical uncertainties have been extrapolated from the Tevatron results, on the basis of the expected ATLAS and CMS detector performances. Combining channels and experiments, LHC should provide a $\mathrm{W}$ mass measurement with a precision beyond the sensitivity of the Tevatron Run2, at the level of $15 \mathrm{MeV}$, in the initial low luminosity phase.

Table 3. Expected contributions to the systematic uncertainty on the W mass measurement in ATLAS for each lepton family and for an integrated luminosity of $10 \mathrm{fb}^{-} 1$. The corresponding uncertainties of the CDF measurement obtained in run1B [14] are shown for comparison

\begin{tabular}{lcc}
\hline Source & $\Delta M_{W}$ CDF Run1B & $\Delta M_{W}$ LHC \\
\hline stat & 65 & $<2$ \\
Lepton scale & 75 & 15 \\
Energy resolution & 25 & $<5$ \\
Recoil model & 37 & 5 \\
Lepton Id. & & 5 \\
$P_{W}^{T}$ & 15 & 5 \\
PDF & 15 & 10 \\
W width & & 7 \\
Radiative decays & 20 & $<10$ \\
Background & 5 & 5 \\
\hline & & $<25$ \\
total $\Delta M_{W}(\mathrm{MeV})$ & 92 &
\end{tabular}

\section{$2.4 \sin ^{2} \theta_{\text {eff }}^{l}$ measurement at the LHC}

$\sin ^{2} \theta_{\text {eff }}^{l}$ can be extracted from the $A_{F B}$ measurement of lepton pairs in the Drell-Yan events. At the $\mathrm{Z}$ pole, the Forward-Backward asymmetry comes from the the interference of the vector and axial components of the coupling, the sensitivity of $A_{F B}$ being parametrized as [15] $A_{F B}=b\left(a-\sin ^{2} \theta_{\text {eff }}^{l}\right)$. The LHC being a p-p collider, the forward direction is not naturally defined. Nevertheles it is still possible to tag the direction of the original quark on a statistical basis. Since the antiquark must be a sea quark, carrying on average a lower momentum than valence quarks, $A_{F B}$ could be signed according to the sign of the rapidity of the lepton pair $y(l l)$. To reach the World Average precision on $\sin ^{2} \theta_{\text {eff }}^{l}$ implies to extend the detection coverage in the forward region, where $A_{F B}$ is significantly larger. A preliminary study, for one LHC 
experiment with $100 \mathrm{fb}^{-} 1$ in the electron channel, has shown [13] that a statistical precision of the order of the WA precision is reachable, extending the pseudorapidity coverage for the second electron up to $|\eta|=4.9$, even with a reduced (factor 100) jet rejection factor. However, a competitive measurement is uncertain, due to the difficulty of controlling the PDF to the required precision, even if new measurements from LHC itself and other experiments will come in the future.

\section{Conclusion}

The most important observables to be measured in the future in the context of the Standard Model will be measured precisely at LHC with ATLAS and CMS. For most of the measurements, and only after one year of running at low luminosity, the precision will be limited by systematic effets, thanks to the large event rate provided by the LHC collider. The Top mass, one of the input parameter used to calculate the prediction of all Standard Model observables will be measured with a precision of the order or less than $1 \mathrm{GeV}$, to be compared to the present reached precision of $5.1 \mathrm{GeV}$. The $\mathrm{W}$ mass precision expected from LHC experiments (of the order of $15 \mathrm{Mev}$ ) should be better than foreseen at Tevatron Run2, mainly because of the large usable samples of $\mathrm{W}$ and $\mathrm{Z}$ decays. Finally, the today world average precision on $\sin ^{2} \theta_{\text {eff }}^{l}$ could be reached if new constraints on PDF are available in the future.

The LHC potential for precision electroweak studies is high. With such expected precision on the Top mass, W mass and $\sin ^{2} \theta_{\text {eff }}^{l}$, the Higgs mass could be constrained with a $20 \%$ relative uncertainty [16]. Nevertheless, detector performances will be crucial to achieve this goal.

\section{References}

1. The LEP Collaborations ALEPH,DELPHI,L3,OPAL, The LEP EW Working Group and the SLD Heavy Flavour Group, 'A Combination of preliminary Electroweak Measurements and Constraints on the Standard Model'. LEPEWWG/2002-01 (2002)

2. The Tevatron EW Working Group and the CDF and D0 Collaborations 'Combination of CDF and D0 results on W Boson Mass and Width' FERMILAB-FN-0716 (2002)

3. The Top averaging Group for the CDF and D0 collaborations 'Combining the Top Quark Mass Results for Run1 from CDF and D0' FERMILAB-TM-2084 (1999)

4. J. Wormesley 'Operation and physics potential of Tevatron Run2' EPJdirect C, 4(S1), 12 (2002)

5. see http://lhc-new-homepage.web.cern.ch/lhc-new-homepage/

6. ATLAS Technical proposal, CERN/LHCC/94-43

7. CMS Technical proposal, CERN/LHCC/94-38 
8. ATLAS Collaboration, Detector and Physics Performance, TDR CERN-LHCC-99-014,CERN-LHCC-99-015 ; ATLAS-TDR-14,15. (1999)

9. P. ROY, PHD thesis Blaise Pascal University, Clermont-Fd, PCCF T 0202 (2002)

10. L.Sonnenschein 'Top mass determination in the $t \bar{t}$ semileptonic decay channel', CMS note 2001/001 (2001)

11. 'Top quark mass measurement with the ATLAS detector at LHC ', ATLAS note ATL-PHYS in preparation (2002)

12. M Beneke et al.: 'Top quark physics',hep-ph/0003033, CERN 2000-004

13. G. Altarelli, M.L. Mangano 'Workshop on standard model physics (and more) at the LHC', CERN 2000-004 (2000)

14. CDF collaboration, hep-ex/0007044 (2001)

15. U.Baur et al. hep-ph/9707301 (1997)

16. The snowmass working Group on Precision Electroweak Measurements, hep-ph/0202001 (2001) 